\title{
Décadrages Décadrages
}

cınéma, à travers champs Cinéma, à travers champs

\section{2-33 | 2016}

\section{Séries télévisées contemporaines}

\section{Apprivoiser le temps, contrôler l'espace : David Claerbout au Mamco}

\section{Achilleas Papakonstantis}

\section{(2) OpenEdition}

\section{Journals}

Édition électronique

URL : http://journals.openedition.org/decadrages/966

DOI : $10.4000 /$ decadrages. 966

ISSN : 2297-5977

Éditeur

Association Décadrages

Édition imprimée

Date de publication : 1 juillet 2016

Pagination : 199-210

ISBN : 978-2-9700963-2-0

ISSN : 2235-7823

Référence électronique

Achilleas Papakonstantis, «Apprivoiser le temps, contrôler l'espace : David Claerbout au Mamco », Décadrages [En ligne], 32-33 | 2016, mis en ligne le 01 décembre 2018, consulté le 01 mai 2019. URL http://journals.openedition.org/decadrages/966 ; DOI : 10.4000/decadrages.966 


\section{Apprivoiser le temps, contrôler l'espace: David Claerbout au Mamco}

LA RÉTrospeCtive QU'A CONSACRÉE le Mamco (Musée d'art moderne et contemporain de Genève) à David Claerbout, du ro juin au iz septembre 20I5, a permis de découvrir plusieurs œuvres de cet artiste belge reconnu depuis le milieu des années I990 dans le champ de l'art contemporain. Son travail imposant et récent nous invite à faire l'expérience d'objets au statut paradoxal, situés aux croisements de la photographie, de l'art vidéo et de l'infographie. Plutôt que de suivre pas à pas les œuvres exposées, nous voudrions insister sur certaines constantes qui les traversent. Ainsi, l'ambition de ce compte rendu n'est pas d'offrir une description linéaire de l'exposition, mais de revenir de manière réflexive sur certaines particularités esthétiques, voire techniques, qui nous semblent marquantes. Nous articulerons un certain nombre d'hypothèses de lecture en inscrivant les œuvres au sein de leurs dispositifs de production et de monstration, en prenant en compte la place et la fonction du spectateur/visiteur.

Cette exposition monographique s’inscrit de plain-pied dans une période caractérisée par l'ouverture de la culture muséale à l'image en mouvement, tendance manifeste également en Suisse et ce de manière de plus en plus fréquente depuis les années 2000. Effectivement, le travail de Claerbout implique certains croisements entre le champ de l'art contemporain et celui du cinéma, au point que d'aucuns le définissent «comme une sortie du cinéma, un après-cinéma, ou encore comme de la photo-vidéo» ${ }^{1}$. Si le titre même de l'exposition, Performed Pictures ${ }^{2}$, suscite des débats nominalistes («images performantes», «art performatif», «happening», etc.), les œuvres elles-mêmes affichent leur caractère conceptuel, s'offrent à la contemplation et donnent tant de prises à l'analyste qu'il ne serait pas exagéré de postuler que leur lieu propre est celui de l'écriture théorique.

A l'instar de Thomas Elsaesser ${ }^{3}$, la plupart des commentateurs de la présence accrue de l'image animée dans les espaces d'art contemporain
1 Elisabeth Chardon, «Exposé à Genève, David Claerbout étire et mêle le temps des images», Le Temps, II juin 2015.

2 Voir la page web officielle de l'exposition, www.mamco.ch/artistes_fichiers/ C/claerbout.html.

3 «[...] le musée n’est pas plus un cinéma qu'un cinéma n'est un musée. Cela tient principalement aux différentes économies temporelles auxquelles il a déjà été fait allusion, et qui obligent le visiteur du musée à <échantillonner > un film plutôt qu'à en faire l'occasion de passer 〈deux heures au cinéma〉. Le temps est ainsi l'une des raisons pour lesquelles le cinéma et le musée constituent deux dispositifs assez distincts, et souvent incompatibles par le passé.» (Thomas Elsaesser, «Entre savoir et croire: le dispositif cinématographique après le cinéma», dans François Albera et Maria Tortajada (éd.), Ciné-dispositifs. Spectacles, cinéma, télévision, littérature, Lausanne, L'Age d'Homme, 20II, p. 6r). 
4 Il est évident que les différences entre "cinéma» et "art contemporain» vont au-delà et en-deçà du moment de la réception actuelle: prédéterminations socioculturelles, relations de pouvoir, politiques de production et de diffusion, autant d'éléments qui exigent d'être pris en compte pour une appréhension globale de la rencontre entre les deux dispositifs.

5 François Albera, «Archéologie de l'intermédialité: SME/CD-ROM, l'apesanteur», Cinémas: revue d'études cinématographiques, vol. I0, $\mathrm{n}^{\circ} 2-3,2000, \mathrm{p} .37$. insistent sur les antinomies fondamentales des dispositifs cinématographique et muséal quant à l'expérience spectatorielle induite, en lien avec l’incompatibilité présumée de leurs configurations spatio-temporelles respectives ${ }^{4}$. Notre hypothèse est que Claerbout propose une nouvelle dynamique entre les deux dispositifs à travers l'économie temporelle de ses «images performantes» et grâce au paradoxe de leur effet immersif. En même temps, ses ouvres vont encore plus loin, jusqu'à la thématisation même de l'expérience spectatorielle: leurs compositions ouvertes et les déambulations de l'œil (effectif ou virtuel) de la caméra modélisent en quelque sorte la flânerie du visiteur des musées. Ainsi, en problématisant l'articulation entre l'espace et le temps par le truchement de la technologie numérique, Claerbout expérimente les seuils de notre perception et pose la question - chargée d'ambiguïtés tant esthétiques qu'idéologiques - du contrôle en nous invitant à interroger les possibilités de création d'un «espace mental de la libre disposition » 5 .

\section{Entre cinéma et installation: vers une nouvelle expérience spatio-temporelle}

D’après le site officiel du Mamco, Performed Pictures est la toute première exposition rétrospective de l'œuvre de David Claerbout. Elle couvre la plus grande partie du parcours de l'artiste: de i996, moment où son esthétique et ses préoccupations théoriques majeures commencent à se cristalliser (notamment par une systématisation du croisement entre la photographie et l'art vidéo), jusqu'à 20I5 et sa toute dernière œuvre, King, achevée peu de temps avant le début de l'exposition. Au total, onze projections vidéo ont été mises à disposition du public, ainsi qu'une collection de photos, des dessins préparatoires et des notes manuscrites de l'artiste offrant un accès privilégié à sa démarche créative. Claerbout prend le plus souvent comme point de départ des images, célèbres ou moins connues, trouvées en bibliothèque, sur internet ou enregistrées par lui-même et ensuite retravaillées (ou littéralement recréées) lors d'un processus de postproduction numérique. En déstabilisant leur statut d'image fixe par l'introduction du mouvement, il met en scène le conflit entre le passé (de la prise de vue) et le présent (de la projection). De la sorte, il nous invite à développer un rapport sémillant avec l'archive et l'histoire; le présent réinjecte du temps (et, dans ce cas précis, du «tempsmouvement») au passé et, de cette manière, le rend actuel. 
L'exemple de Ruurlo, Bocurloscheweg, I9I0 (I997) est éloquent. L'image-source est ici une photographie datant du début du $\mathrm{XX}^{\mathrm{e}}$ siècle représentant un arbre derrière lequel sont visibles un moulin à vent et quelques passants. A première vue, tout semble figé dans une immobilité absolue. Et pourtant, en regardant l'image suffisamment longtemps, le spectateur aperçoit finalement le mouvement quasi imperceptible du feuillage de l'arbre, «un frémissement atmosphérique numériquement introduit dans la photographie d'une scène ancienne» 6 . Une série de questions surgissent. Est-ce que l'arbre est transposé d'un autre lieu grâce à un montage qui - en nous rappelant les écrits de Koulechov - produit des espaces géographiques et plastiques (ici à l'intérieur d'un seul cadre) qui n'ont jamais existé auparavant en tant que tels? S’agit-il d'une nouvelle synthèse spatio-temporelle par l'introduction d'un objet mouvant d'après et d'ailleurs? Un élément paratextuel, à savoir le côtoiement de deux dates dans le titre (I9I0-I997), met l'accent sur la réactivation du passé par le présent qu’on pourrait également appréhender à un niveau technologique - en interprétant, par exemple, la rencontre entre photographie analogique et montage numérique, ou encore entre photographie et cinéma, dans la logique de l'archéologie des médias. Par ailleurs, on ne peut manquer de voir une référence au passé technique et à l'imaginaire des origines de l'image en mouvement: on le sait, ce n'était pas tant la présence des acteurs captés sur le vif qui avait impressionné les premiers spectateurs du cinématographe Lumière, mais plutôt le léger mouvement du feuillage de l'arbre à l'arrière-plan dans le Repas de bébé.

L'objectif principal de Claerbout se présente comme l'instauration d'un rapport autre au temps, voire comme la construction d'un temps plastique, étranger à nos habitudes perceptives et socioculturelles. Il expérimente sur les possibilités de visualisation de la durée pure, soit par l'emploi d'une dilatation exacerbée (voir par exemple Oil Workers, où la perception du temps est liée à la lenteur du défilement des images), soit par la longueur extrême de l'œuvre projetée (voir Bordeaux Piece d'une durée totale de quatorze heures). Mais le temps peut être appréhendé ici également comme un paramètre spatial. Long Goodbye (2007) constitue un cas exemplaire de cette configuration en conférant une fonction double aux ombres: preuve de l'existence matérielle des corps et des objets représentés et, in extenso, de l'image elle-même, elles incarnent pardessus tout la représentation, tangible mais inexorablement fugace, du
6 Thierry Davila, Shadow Pieces (David Claerbout), Genève, Editions Mamco, 2015 , p. I8. 


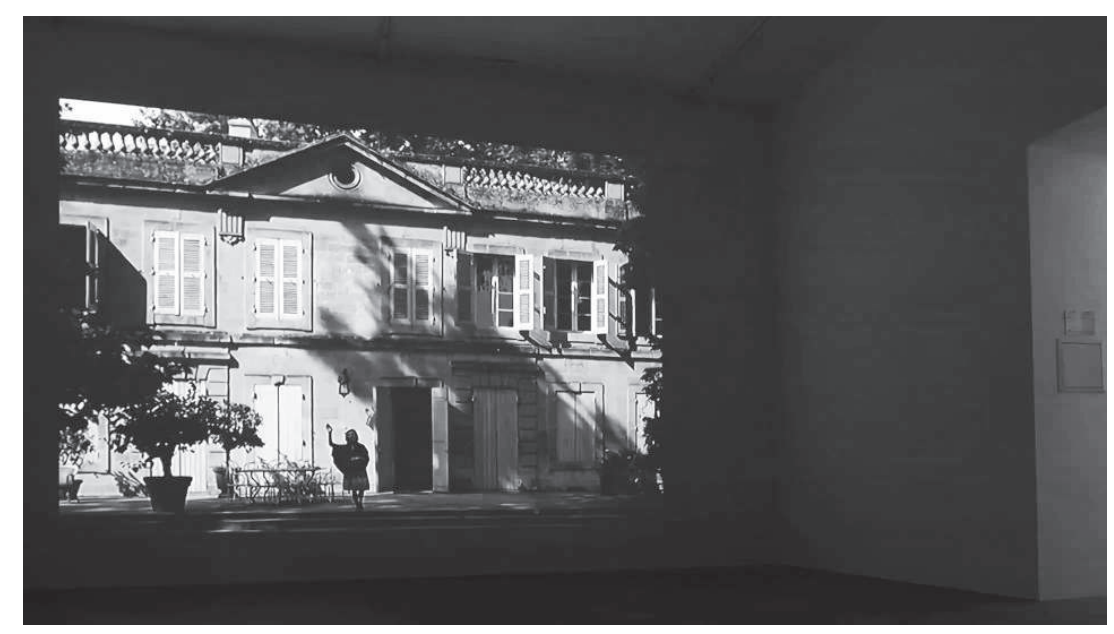

temps qui passe. L'ombre est la durée visible; mais il s'agit d'une durée manipulée, construite et hétérogène. En effet, plusieurs temps coexistent à l'intérieur de ce «plan-séquence». Une femme sort d'une maison, se dirige vers une table à une terrasse et verse du thé tout en dévisageant la caméra (et, donc, le spectateur). Sa main se lève et s'agite doucement dans l'air pour (nous) dire au revoir. Ses mouvements sont capturés par un travelling arrière projeté au ralenti. Or, un sentiment d'étrangeté spatio-temporelle se produit par le parcours extrêmement rapide d'ombres qui fonctionnent comme des repères reconnaissables de l'itinéraire du soleil et qui signalent ainsi le passage d'une journée entière dans le laps de temps de douze minutes (la durée totale de projection de l'œuvre). Larrivée de la nuit forme un fondu au noir naturel et annonce la fin du «film». Il est question ici d'un morcellement de la dimension temporelle, de sa division, qui permet à un seul plan d'accueillir à la fois son ralentissement et son accélération ( fig. I).

Cette œuvre remarquable se distingue également pour des raisons plastiques. Sa composition équilibrée propose un chassé-croisé formé par le travelling en arrière de la caméra et le défilé latéral (de gauche à droite) des ombres - deux mouvements qui ont lieu simultanément dans l'espace de la représentation tout en se déployant dans des univers tem- 
porels distincts. En outre, la lenteur excessive du déplacement du personnage féminin produit une esthétisation de sa gestuelle. Or, Long Goodbye s'ouvre sur un cadre qui renvoie aux portraits de la peinture flamande: un gros plan sur le visage de la femme, vu de trois quarts et entouré de noir. Quelques secondes sont nécessaires avant de percevoir son mouvement et, grâce à l'élargissement graduel du cadre, d'attribuer l'obscurité ambiante à l'intérieur de la maison qui contraste avec le soleil brillant de l'espace extérieur. La référence picturale renvoie de nouveau au principe d'introduction du mouvement à l'intérieur d'une image fixe, érigé en matrice organisationnelle de la pratique artistique de Claerbout. Le tableau prend vie et se trouve désormais hanté par la présence fantomatique de la femme, dont la figure semble superposée sur l'espace, venue d'un monde différent. Mais cette ouverture vers un ailleurs et un autrefois se produit à la fois en amont et en aval du geste créatif: le regard-caméra de la femme fait appel au regard du spectateur (qui incarne alors l'autre de la représentation) et, ainsi, le champ convoque le hors-champ. Cette représentation interpellait d'autant plus le visiteur de l'exposition au Mamco par la scénographie qui permettait de voir l'écran de loin, depuis une autre salle et à travers l'encadrement d'une porte. Force est de constater que ce dispositif produit un type de spectateur particulier: un corps qui observe et se perçoit, transformé en agent actif dans une configuration ouverte et dynamique.

Ce schéma actanciel formé par la représentation, le spectateur et la machinerie (au sens large du terme, qui inclut les caractéristiques architecturales des salles ainsi que les choix de «mise en scène» privilégiés par les organisateurs de l'exposition) devient encore plus saillant dans Shadow Piece (2005). Claerbout a conçu cette «pièce» d'après une photo d'archive anonyme. Placée sur l'escalier à l'intérieur d'un immeuble, la caméra cadre en plongée une série de portes en verre qui forment l'entrée du bâtiment. La source de lumière se situe dans le hors-champ, au-delà des portes, à l'extérieur de l'immeuble. De temps à autre, un passant tente en vain d'ouvrir la porte; c'est seulement son ombre qui réussit à traverser la barrière transparente formée par la masse vitreuse. Le cadre est divisé en deux parties. En haut, on aperçoit les figures des passants qui défilent dans un mouvement incessant; en bas, la salle vide semble condamnée dans une fixité lugubre. Les ombres, véritables présences fantomatiques, glissent de haut en bas, de l'extérieur à l'intérieur, et 
7 On remarque ici le rôle du hasard qui influe sur l'expérience spectatorielle: le chemin qu'on emprunte lors de notre visite à l'exposition a des répercussions sur notre rencontre avec le dispositif de Shadow Piece. émergent comme l'intrication matérialisée entre le mouvement et l'immobilité (et, selon une autre hypothèse de lecture, entre le cinématographique et le photographique représentés respectivement par les parties supérieure et inférieure du cadre). Comme on l'a déjà remarqué à propos de Long Goodbye, le jeu des ombres permet de visualiser la durée et d'inoculer du temps à une image du passé (fig. 2).

Toutefois, Shadow Piece est une œuvre qui s'actualise à travers le spectateur. Projetée sur une toile blanche accrochée au plafond de sorte que la salle se divise en deux parties (devant et derrière l'écran), la représentation s'ouvre à l'espace de l'exposition. Le visiteur qui, lors de son itinéraire, entre dans la salle par la porte arrière ${ }^{7}$, s'introduit à son insu dans le cadre et sa silhouette ténébreuse défile aux côtés des ombres filmées auparavant par la caméra de Claerbout. Ainsi, l'enchevêtrement de deux temporalités advient ici à un niveau supplémentaire, par l'interpénétration de l'espace de la représentation et de celui de la réception. Une sorte de spectatorialité collective émerge de ce dispositif puisque le visiteur, en plus d'observer l'œuvre et de se percevoir, perçoit également les autres visiteurs dans l'ici et maintenant de leur coprésence dans

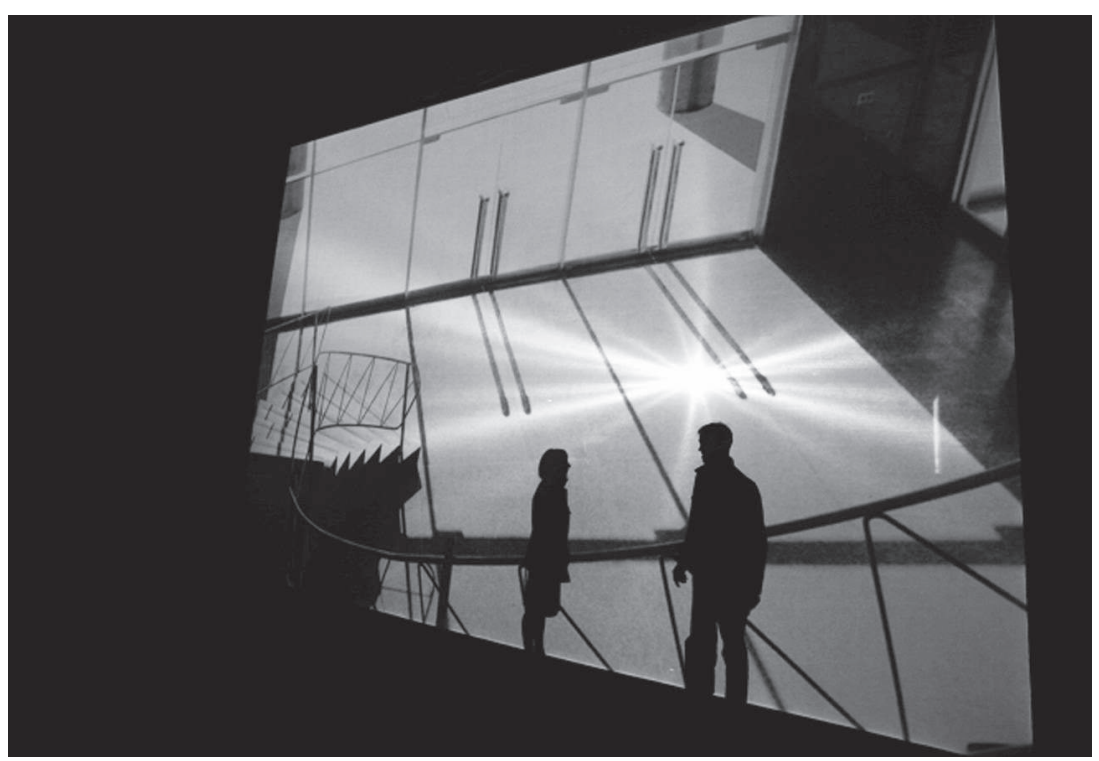


le musée ${ }^{8}$. La flânerie n'est pas seulement thématisée, elle s'intègre à la représentation.

La porosité des frontières séparant l'expérience du visiteur du musée et celle du spectateur du cinéma est explicitement étudiée dans Travel (1996-2013). Selon Claerbout ${ }^{9}$, cette œuvre ne remonte pas à une image d'archive mais à une mélodie, un morceau écrit par Eric Breton, spécialiste de la musique thérapeutique, censé produire la détente de son auditeur, allant même jusqu'au sommeil. De la même manière, l'idée derrière Travel est de créer un court métrage susceptible de relaxer son spectateur et, dans l'idéal, l'endormir ${ }^{10}$. L'effet immersif déjà présent dans Long Goodbye, Oil Workers, Ruurlo, Bocurloscheweg, ıgıo et même Shadow Piece (dû chaque fois au ralenti hypnotisant, à la démultiplication des univers temporels ou à d'autres stratégies visant à exproprier le spectateur de ses repères perceptifs habituels) se trouve ici exacerbé. Au début de la projection, nous nous trouvons à l'entrée d'un parc. Ensuite, dans un mouvement continu et ininterrompu, la caméra virtuelle de Claerbout s'enfonce dans une forêt qui s'avère être une jungle amazonienne, onirique et paisible et, enfin, sortant de la forêt par un mouvement ascendant, révèle une plaine située dans une banlieue assez banale. Par la construction numérique de ce paysage (car il s'agit bien d'une image de synthèse), Claerbout ne vise pas à une beauté à couper le souffle (d'ailleurs on a pu vérifier en lisant les notes manuscrites de l'artiste exposées au Mamco, que l'esthétique kitsch de l'œuvre a été tout à fait délibérée); son ambition a été plutôt de construire une visualisation de l'idée générique de «forêt», universellement partagée, comme un abri tranquille et serein, un refuge destiné à nous relaxer.

Mais l'effet immersif n'est pas produit par le seul aspect représentationnel de l'œuvre. Travel est projeté dans une petite chambre aménagée en salle de cinéma, un espace isolé brisant en quelque sorte la continuité d'une exposition d'installations à parcourir. Qui plus est, une série de fauteuils, dont la forme garantit un maximum de confort, est rangée devant l'écran. L'immersion spectatorielle résulte également d'une organisation temporelle particulière. Accroché sur le mur, à côté de l'entrée de cette salle, se trouve un moniteur qui annonce par une horloge faisant le compte à rebours le début de la prochaine projection (l'œuvre, d'une durée totale de douze minutes, était projetée en boucle avec un intervalle d'environ cinq minutes entre chaque «séance»). En franchissant le seuil
8 D'ailleurs, cette configuration est susceptible de provoquer une tension temporelle chez le spectateur. Une fois qu'on a deviné le jeu, on est incité à rester devant l'écran et à attendre qu'un autre visiteur arrive derrière la toile et nous offre le spectacle de son ombre.

9 Voir notamment les commentaires sur le site officiel de l'artiste: www.david claerbout.com/Travel-I996-2013.

10 «Je me suis dit que créer une œuvre qui pourrait endormir le public n’était pas une si mauvaise chose» [notre trad.], $i d$. 
11 Thomas Elsaesser, «Entre savoir et croire: le dispositif cinématographique après le cinéma», op. cit., p. 62.

12 Rappelons-nous qu'un des effets fondamentaux de toute image en mouvement est la formation d'un sujet percevant pour qui la continuité entre l'espace fictionnel et l'espace réel est à la fois implicite et systématiquement dénié. de la porte à l'heure annoncée et en prenant sa place dans ces fauteuils extrêmement confortables, le spectateur accepte un contrat de lecture très précis, s’immerge dans un monde fictif et s'oublie, rendant ainsi impossible la forme de présence que l’institution muséale a érigé en norme: «un 〈moi , un 〈ici〉 et un 〈maintenant`» "11, une conscience spatiotemporelle, voire une sensibilité d'ordre phénoménologique envers tout ce qui est présent (y compris son propre corps). En revanche, nous nous trouvons ici à l'intérieur d'une configuration qui renvoie à une séance de cinéma dans sa forme institutionnalisée: salle obscure, image animée, spectateur immobile. Claerbout pousse à l'extrême l'effet immersif du spectacle cinématographique et se présente comme un agent provocateur qui base son jeu sur la tension produite par la présence de l'image en mouvement dans l'espace de la galerie ${ }^{12}$.

\section{La technologie numérique au service d'une didactique du regard}

Si l'exposition dans son ensemble met l'accent sur les différentes modalités de sollicitation de l'espace de réception, The Algiers' Sections of a Happy Moment (2008) constitue la manifestation la plus audacieuse de ce principe. Ce slide show d'une durée totale de trente-sept minutes se présente comme un défilé fluide de multiples vues fixes. Au premier regard, on a l'impression d'assister à un seul instant, photographié simultanément depuis des points de vue différents et extrêmement nombreux qui se succèdent au cours de la projection: sur un terrain situé en plein air dans la Casbah d'Alger, de jeunes garçons jouent au football tandis qu'à côté d'eux, un homme lance des morceaux de pain à une nuée de mouettes qui volent tout autour. A une première image générale, la seule qui donne accès à une vue d'ensemble de la scène, succède un montage de plans fixes qui se livre à une analyse «multifocale» de la scène principale. Cette démarche de mise en mouvement d'une image fixe par découpes successives n'a rien d'original en soi. Reste à comprendre comment Claerbout est parvenu à saisir un instant unique depuis cinq cents - le nombre d'images qui composent l'œuvre - points de vue différents ( fig. 3).

Lorsqu'on observe attentivement l'espace de la représentation, l'on aperçoit plusieurs traces d'une dissonance visuelle, autant de signes d'une superposition a posteriori des figures humaines sur le terrain. En effet, The Algiers' Sections of a Happy Moment s'avère être le résultat d'un travail en studio. Les différentes vues ont été enregistrées séparément, à 


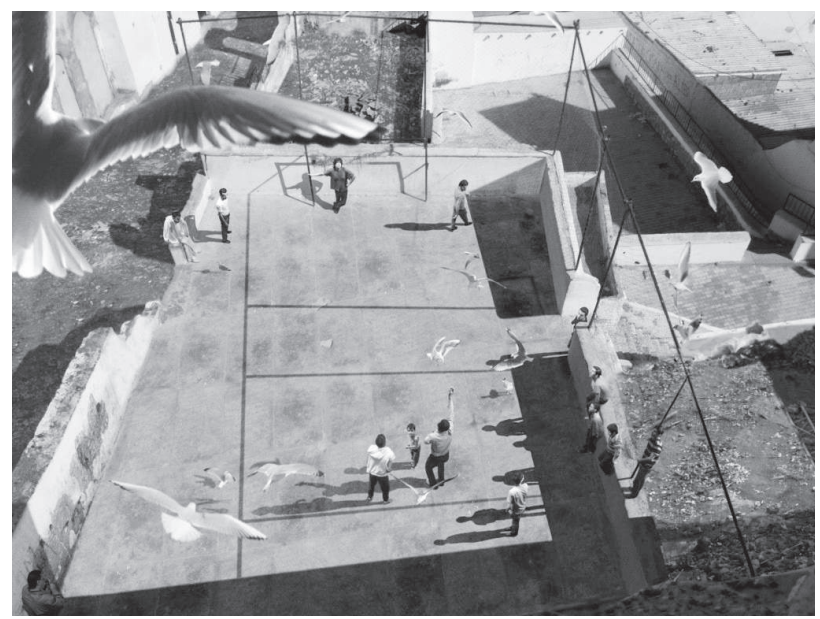

des moments différents, avant d'être réarrangées par le biais d'une postproduction numérique pour composer la scène globale. Comme dans Long Goodbye, mais ici de façon plus ambitieuse, on fait face à un instant construit, synthèse de plusieurs temps différents. Les personnages de ce «film» proviennent de mondes profilmiques ${ }^{13}$ qui, en eux-mêmes, ne communiquaient pas. Claerbout les rapproche et leur fait entretenir un rapport de coréférence spatiale par le biais d'une incrustation numérique. Ce dispositif très particulier nous invite à l'exploration de tous les recoins d'une scène à la faveur d'un déblocage au niveau de l'angle de prise de vue. De cette manière, il met en péril cette fonction primordiale du cadre photographique qui consiste à déterminer les limites de l'image et celles du regard spectatoriel. L'espace représenté n'est plus clos et circonscrit et ne se termine pas sur les bords du cadre; il se dissout et, qui plus est, se dissout dans le temps (à savoir le temps de projection). Ainsi, The Algiers' Sections of a Happy Moment se présente comme une tentative de dépassement des limitations spatiotemporelles imposées par le cadre. Il s'agit bien d'une didactique: la technologie numérique nous permet de déjouer la frustration de notre regard dont le désir d'omnipotence se voit systématiquement refoulé dans la vie quotidienne. Claerbout exploite les potentiels expressifs et techniques des procédés digitaux pour nous faire voir plus et mieux ${ }^{14}$.
3/ The Algiers' Sections of a Happy Moment (2008)

13 On entend le profilmique comme «ce qui s'est trouvé devant la caméra au moment du tournage, que cela y ait été disposé intentionnellement ou non» (Jacques Aumont et Michel Marie, Dictionnaire théorique et critique du cinéma, Paris, $\mathrm{Na}$ than, 200I, p. I66).

14 L'idée de la technologie numérique comme «machine» qui permet d'accroître les possibilités de l'œil humain nous invite à faire le lien avec les réflexions des avant-gardes artistiques des années 1920 sur le cinéma et la photographie. 
Même s'il met en place un dispositif favorisant une perception à la fois englobante et diffractée, Claerbout prend pourtant soin de ne pas excéder la capacité d'absorption du spectateur et ceci grâce à un défilement des images lent et fluide, étayé par le choix du fondu enchaîné comme modalité de transition; dans le même temps, la musique hypnotisante qui accompagne la projection contribue à un effet immersif renvoyant plutôt à une séance de cinéma. Pour revenir à la problématique qui a embrayé jusqu'ici notre réflexion, on pourrait interpréter ce qu’on voit sur l'écran comme un discours sur les différents modes de spectatorialité normalisés par les institutions cinématographique et muséale. The Algiers' Sections of a Happy Moment met en abîme et thématise l'activité du visiteur des galeries, sa flânerie déployée en trois dimensions lui permettant d'explorer les œuvres exposées de différents points de vue. En laissant de côté les connotations métaphysiques (l'idée d'une omnivoyance qui renvoie à l'œil divin), cette exégèse de l'œuvre comme symbolisant l'activité spectatorielle nous renvoie à la question du contrôle. L'élimination des angles morts et l'idée d'un épuisement des points de vue donnent au spectateur une sensation de puissance, de maîtrise de la scène représentée, comme s'il se trouvait dans une salle de contrôle d'images de surveillance. Nous sommes ainsi tentés de faire le lien entre ce dispositif de vision qui donne l'illusion d'une émancipation du regard et l'imaginaire d'omni-perception, un fantasme qui entoure précisément la diffusion massive de la technologie numérique. Cependant, dans The Algiers' Sections of a Happy Moment, l'idée d'une toute-puissance perceptive qui offre un accès privilégié à l'instant saisi n'est qu'un leurre. On n'a pas affaire à un instant, mais à une multitude d'instants différents combinés par une synthèse préconçue et fabriquée. Par ailleurs, le contrôle du perçu promis au spectateur exige son propre effacement et son immersion dans l'espace de la représentation - comme dans Travel, la tension entre spectateur de cinéma et visiteur de musée est ici explicitement travaillée par l'artiste.

Dans son œuvre exposée la plus récente, King (2015), Claerbout poursuit l'élaboration d'un mode de perception considéré comme spécifique à l'ère du numérique. Cette fois, l'image qui sert de point de départ jouit d'une bonne réputation: c'est une photographie d'Elvis Presley en 1956 , signée par Alfred Wertheimer et souvent interprétée comme le symbole de la transition du «King» de la vie ordinaire à la célébrité. Claerbout 


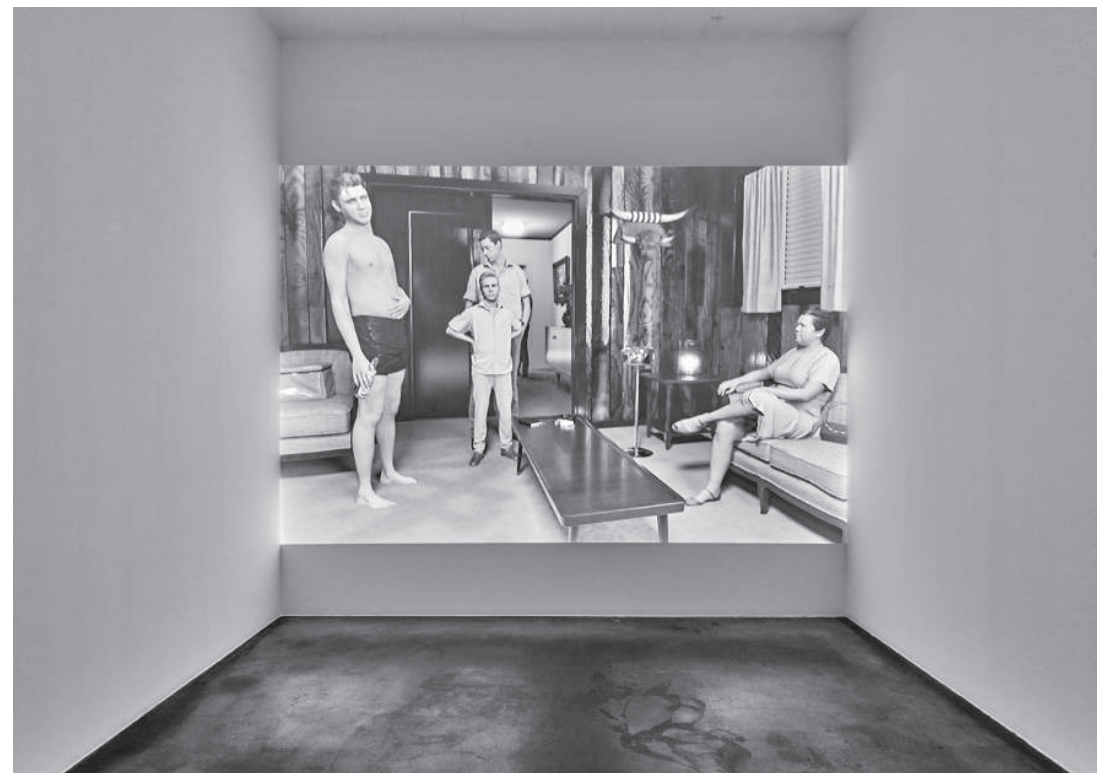

procède à une récréation numérique de cette photo, une animation en $3 \mathrm{D}$ où la figure d'Elvis est composée des fragments de centaines de photographies originales de la star. En poussant plus loin la logique de The Algiers' Sections of a Happy Moment, on se porte encore une fois à l'encontre de la limitation bidimensionnelle du cadre photographique, mais cette fois sans avoir recours à une succession de plans; dans un mouvement continu (un «plan-séquence» projeté en boucle), une caméra virtuelle nous permet de nous rapprocher d'Elvis, de tourner autour de lui et de porter notre regard sur tout ce qui se trouvait dans la chambre mais a été cantonné dans le hors-champ par le geste photographique original. Ainsi, la technologie numérique sculpte le paysage d'une image ancienne dont l'espace représenté se transforme désormais en univers malléable. Toutefois, la «réincarnation» digitale de ce corps sacré ne cherche pas à être réaliste ou transparente. Les imperfections de la synthèse numérique sont manifestes à ce point que la matérialité de l'image (les pixels) finit par attirer l'attention du spectateur. En même temps qu'on nous donne la possibilité de scruter de tout près la figure mythique 
15 Bordeaux Piece constitue la manifestation la plus extrême de ce principe. Claerbout mobilise le savoir intertextuel des spectateurs en employant la trame narrative d'un des films les plus connus de Jean-Luc Godard, Le Mépris (1963). Mais l'histoire n'est ici qu'un simple prétexte: «C'est la lumière qui organise tout. On peut s'intéresser au récit la première fois, peut-être la seconde, mais déjà il devient une sorte de canevas assez décevant, un motif rythmant le véritable enjeu de Bordeaux Piece, qui est de donner une forme à la durée au moyen de la lumière naturelle» (David Claerbout dans Marie Muracciole, «La bruit des images. Conversation avec David Claerbout», Cahiers du Musée nation d'art moderne, $\mathrm{n}^{\circ} 94$, hiver 2005-2006, pp. I25-126).
d'Elvis, le processus de fétichisation est nié par une décomposition de l'illusion référentielle, propre à la réalité virtuelle (fig. 4).

\section{Pour une émancipation politique du temps}

L'exposition au Mamco a confirmé David Claerbout comme l'une des figures de proue de la réflexion sur l'image digitale. En dédramatisant l'aspect représentationnel de ses œuvres, l'artiste se met à la recherche d'une esthétique propre à la technologie numérique. Il invite le spectateur/ visiteur à explorer le langage des médias digitaux dont l'«impureté» immanente aménage un espace intermédial, entre le cinéma et la photographie, ou encore entre l'installation et les arts ciné-plastiques. Il retient du spectacle cinématographique son effet immersif, mais il écarte son obstination narrative, la primauté traditionnellement donnée à l'histoire. En effet, dans toutes les œuvres de Claerbout proposées au public genevois, l'«événement raconté» se retire vers l'arrière-plan et laisse sa place au récit du temps qui passe ${ }^{15}$. Toutefois, pour faire l'expérience de la durée pure, le spectateur doit abandonner ses acquis socioculturels (voire idéologiques) et, notamment, son idée du temps comme une valeur calculée et convertible en argent comptant. L'art de Claerbout nous invite à (re)donner à la vie son épaisseur et la rendre ainsi justiciable d'une expérience esthétique.

Ainsi, dans Venice Lightboxes, on se trouve face à quatre vues nocturnes de Venise (le palais de Ca'd'Oro et les églises d'Isola San Michele, Santa Maria della Salute et Isola San Giorgio), des clichés pris entre quatre et six heures du matin et exposés dans une salle peinte en noir et plongée dans l'obscurité. Le visiteur doit rester assez longtemps dans cette «salle obscure» pour que son œil s'habitue à la nuit double - celle figurée dans la représentation et celle induite par la machinerie du dispositif. Après quelques minutes, le vide commence à être habité, l'image fait corps avec le spectateur et une impression de mouvement se produit. En un geste, Claerbout déconstruit le caractère spectaculaire des monuments systématiquement exploités par les institutions culturelles et touristiques, délivre le visiteur de son regard codé socialement et culturellement et propose une autre manière de voir dans le temps, de voir le temps et, de là, d'être au monde. 